Polibotánica

ISSN electrónico: 2395-9525

POLIBETÁNICA

polibotanica@gmail.com

Instituto Politécnico Nacional

México

http:www.polibotanica.mx

\author{
VEGETACIÓN Y FLORA \\ FANEROGÁMICA DEL ÁREA \\ NATURAL PROTEGIDA PIEDRAS \\ BOLA, JALISCO, MÉXICO

\section{VEGETATION AND \\ PHANEROGAMIC FLORA OF \\ PIEDRAS BOLA PROTECTED AREA \\ IN JALISCO, MEXICO}

García-Martínez, M.A., y A. Rodríguez

VEGETACIÓN Y FLORA FANEROGÁMICA DEL ÁREA NATURAL PROTEGIDA PIEDRAS BOLA, JALISCO, MÉXICO.

VEGETATION AND PHANEROGAMIC FLORA OF PIEDRAS BOLA PROTECTED AREA IN JALISCO, MEXICO.

POLIBETÁNICA

Instituto Politécnico Nacional
Núm. 46: 71-90 México. Julio 2018

DOI: $10.18387 /$ polibotanica.46.4 


\section{VEGETACIÓN Y FLORA FANEROGÁMICA DEL ÁREA NATURAL PROTEGIDA PIEDRAS BOLA, JALISCO, MÉXICO}

García-Martínez, M.A., y A. Rodríguez

VEGETACIÓN Y FLORA FANEROGÁMICA DEL ÁREA NATURAL PROTEGIDA PIEDRAS BOLA, JALISCO, MÉXICO

VEGETATION AND

PHANEROGAMIC FLORA OF PIEDRAS BOLA

PROTECTED AREA IN

JALISCO, MEXICO

POLIBETÁNICA Instituto Politécnico Nacional

Núm. 46: 71-90. Julio 2018

DOI:

10.18387/polibotanica.46.4
M.A. García-Martínez/mtz.migl@gmail.com Centro Universitario de Ciencias Biológicas y Agropecuarias. Apartado postal 1-139, Zapopan 45101, Jalisco, México.

A. Rodríguez/ aaron.rodriguez@cucba.udg.mx Herbario Luz María Villarreal de Puga del Instituto de Botánica de la Universidad de Guadalajara (IBUG), Departamento de Botánica y Zoología. Apartado postal 1-139, Zapopan 45101, Jalisco, México.

RESUMEN: El Área Natural Protegida Piedras Bola (ANPPB) fue nombrada y decretada por la existencia de rocas esféricas de hasta $1.5 \mathrm{~m}$ de diámetro. Estas rocas tienen un origen volcánico y su formación representa un fenómeno poco común. El área se ubica en el estado de Jalisco, sobre la porción oeste de la Faja Volcánica Transmexicana. El bosque de Quercus es la vegetación dominante seguida del bosque mixto de Quercus y Pinus. El objetivo del presente estudio fue realizar el inventario de las plantas fanerógamas del ANPPB. Para tal efecto, se realizaron 24 exploraciones entre 2011 y 2016 . En total se registraron 54 familias, 170 géneros y 262 especies. Las familias mejor representadas fueron Asteraceae (47 spp.), Fabaceae (40 spp.), Poaceae (28 spp.) y Solanaceae (15 spp.). Los resultados se comparan con estudios previos realizados en el ANPPB y con inventarios florísticos de áreas cercanas que cuentan con condiciones ecológicas similares.

Palabras clave: Ahualulco de Mercado, Faja Volcánica Transmexicana, rocas esféricas, Sierra del Águila, Sierra de Ameca.

ABSTRACT: The Piedras Bola Natural Protected Area (ANPPB) was named and decreed due to the presence of stone spheres in the area which can reach up $1.5 \mathrm{~m}$ in diameter. These stones have a volcanic origin and their formation represents an uncommon phenomenon. The area is located in the state of Jalisco, in the western portion of the Transmexican Volcanic Belt. The dominant vegetation type is the oak forest and oak-pine forest. The aim of the present work was to make an inventory of the phanerogamic flora of the area. We carried out 24 explorations between 2011 and 2016 and we enlist 54 families, 170 genera and 262 species. The most representative families were Asteraceae (47 spp.), Fabaceae (40 spp.), Poaceae (28 spp.) and Solanaceae (15 spp.). The results were compared with previous work in the Piedras Bola area and with floristic studies carried out in nearby areas with similar ecological conditions.

Key words: Ahualulco de Mercado, Sierra del Águila, Sierra de Ameca, stone spheres, Transmexican Volcanic Belt. 


\section{INTRODUCCIÓN}

El Área Natural Protegida Piedras Bola (ANPPB) fue decretada en el año 2007 dentro de la categoría de Zona de Conservación Ecológica Municipal, con la clasificación local de Formaciones Naturales de Interés Municipal (Gobierno de Jalisco, 2007). El nombre y la razón por la que se ha decretado esta área natural se debe a la existencia de rocas esféricas ígneas extrusivas, también conocidas como megaesferulitas. El diámetro de estas rocas varía entre 0.9 a $1.5 \mathrm{~m}$ y su origen se debe a un fenómeno inusual. Existen diversas hipótesis sobre su formación (Curiel-Ballesteros, 1997; Stirling, 1969). La propuesta más reciente describe que su formación inició con una corriente piroclástica a lo largo de un valle fluvial estrecho. La dirección del flujo y la pendiente del movimiento fueron constantes durante el enfriamiento y aglomeración de los piroclastos (Castillo-Girón y Aceves-Ávila, 2007). Se han registrado 74 rocas, en algunos casos se aprecia su cuerpo completo, en otros, el suelo las cubre parcialmente. Existe un caso especial, donde el intemperismo ha formado un pedestal de $3.5 \mathrm{~m}$ de altura sobre el cual yace una roca. Esta formación se conoce como Las Torrecillas (fig. 1A). Las rocas esféricas son el atractivo principal de la región. El valor geológico, ambiental, social y económico de la zona es elevado y poco apreciado. En conjunto con el paisaje montañoso, cubierto en su mayoría por encinar, las Piedras Bola lucen como parte del patrimonio cultural de los habitantes del municipio de Ahualulco de Mercado y de la región (fig. 1).

A pesar de su importancia, el ANPPB no cuenta con un inventario biológico formal. Por esta razón, el H. Ayuntamiento Constitucional de Ahualulco de Mercado administración 2010-2012, realizó un vínculo con el Centro Universitario de Ciencias Biológicas y Agropecuarias (CUCBA) de la Universidad de Guadalajara para realizar el inventario biológico del área.

Existen dos trabajos previos realizados en la zona. Curiel-Ballesteros (1997) realizó el primer esfuerzo para proponer la protección del área de Piedras Bola. En el trabajo referido fue realizada la caracterización biótica y abiótica del área. En él se presenta un listado florístico, pero no se indica el método de muestreo. Por su parte, Contreras-Rodríguez et al. (2000) realizaron un estudio ecológico, con el objetivo de determinar la frecuencia, densidad, dominancia e índice de importancia para las especies muestreadas. De igual forma presenta un listado, pero no se indica la ubicación y el número de transectos realizados. Por consiguiente, los objetivos del presente trabajo son 1) realizar el inventario de las plantas fanerógamas del ANPPB , 2) comparar los resultados con estudios previos realizados en el ANPPB y 3) realizar una comparación con inventarios florísticos realizados en áreas cercanas que cuenten con tipos de vegetación similares, utilizando el índice de diversidad taxonómica.

\section{MÉTODOS}

\section{Área de estudio}

El ANPPB tiene una superficie de 256 hectáreas que son parte del terreno de uso común de 49 ejidatarios en el Ejido de Santa Cruz de Bárcenas, municipio de Ahualulco de Mercado. El intervalo de elevación va de los 1820 a los $2080 \mathrm{~m}$. El ingreso a la zona protegida se ubica en el km 14.1 del camino vecinal entre Ahualulco de Mercado y Ameca (fig. 2). A partir de este punto, existe una brecha de casi $5 \mathrm{~km}$ para llegar hasta la zona de esferas y el campamento del Área Natural Protegida ubicados a una altitud de 1937 m, en el paralelo $20^{\circ} 39^{\prime} 15.98^{\prime \prime}$ de latitud Norte y el meridiano $104^{\circ} 03^{\prime} 28.62^{\prime \prime}$ de longitud Oeste. 

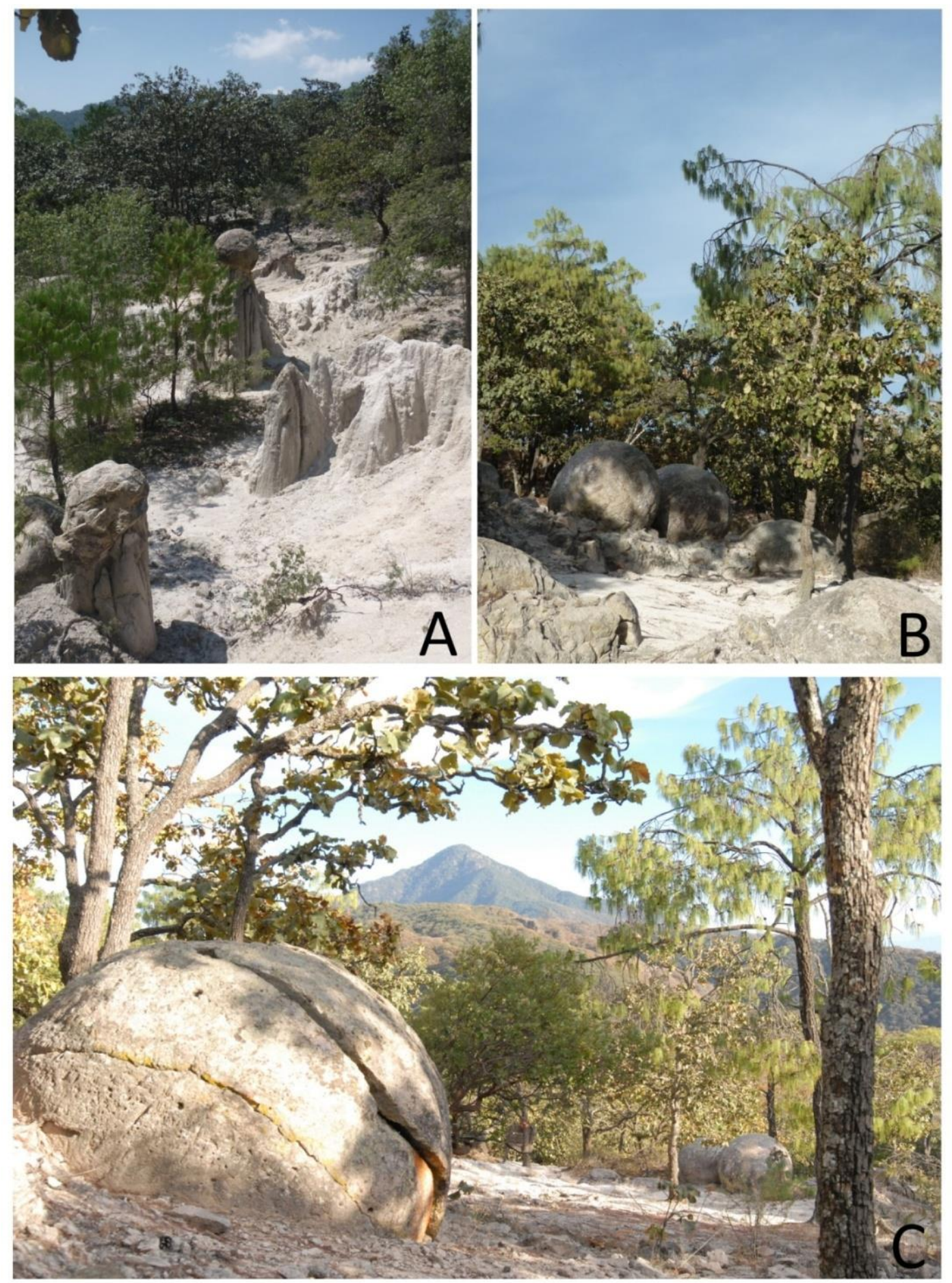

Fig. 1: Paisaje característico del Área Natural Protegida Piedras Bola y formaciones de rocas esféricas que dan el nombre a la zona. A. Formación conocida como Torrecillas. B. Piedras Bola de $1.5 \mathrm{~m}$ de diámetro. C. Vista general del bosque mixto de Quercus y Pinus y al fondo se aprecia el Cerro del Águila (Fotografías de Rodríguez, A.). 


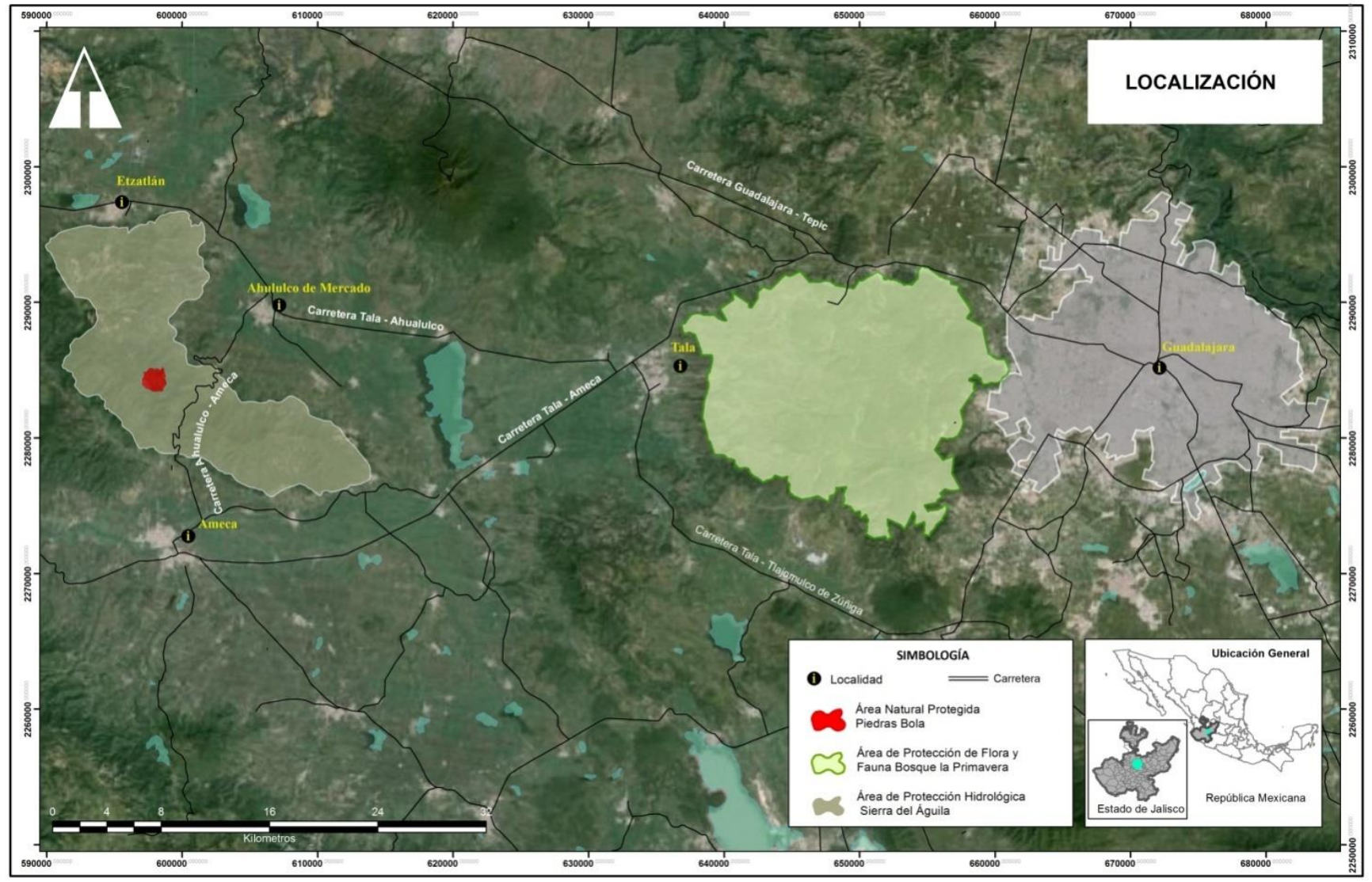

Fig. 2: Localización del Área Natural Protegida Piedras Bola. En el mapa se señalan las principales localidades y carreteras. El ANPPB es parte de la Sierra del Águila.

Fisiografía: El ANPPB pertenece a la Faja Volcánica Transmexicana (FVT). La FVT es un conjunto de mesetas escalonadas y volcanes alineados sobre una franja que cruza el territorio mexicano desde el Parque Nacional Cerro de San Juan, en Nayarit, hasta la Sierra de Chinconquiaco, en Veracruz (Espinosa y Ocegueda, 2007; Lugo-Hubp, 2004). En Jalisco, una formación montañosa conocida como Sierra del Águila, decretada como Área Estatal de Protección Hidrológica (Gobierno de Jalisco, 2010), se encuentra definida y limitada por los valles y zonas agrícolas de Ahualulco de Mercado, Etzatlán y Ameca. El ANPPB es parte de la Sierra del Águila (fig. 2).

Geología: En el ANPPB se encuentran dos tipos de rocas ígneas de la era Cenozoica. En la porción suroeste se registra el basalto, mientras que en la porción noreste del polígono se encuentra un macizo de toba ácida (INEGI, 2017).

Edafología: Con respecto al tipo de suelo, solo se registra el leptosol. Este es el tipo de suelo más común a nivel mundial y se encuentra asociado a sitios con orografía compleja. Se caracterizan por ser delgados, pedregosos y poco desarrollados. En su composición se encuentran cantidades significativas de material calcáreo. Este mineral puede inmovilizar los nutrientes minerales del suelo por lo que su uso agrícola es limitado (INEGI, 2017; SEMARNAT, 2013). Esta característica del suelo ha favorecido la conservación del área.

Hidrología: El ANPPB es parte de la cuenca del río Ameca. Cerca de una cuarta parte de su superficie drena hacia el norte en el valle de Ahualulco. Posteriormente, el agua se encauza 
hasta la presa de La Vega. El resto de la zona drena hacia el sur, en el valle de Ameca. En donde el agua se incorpora al arroyo del poblado El Arco y eventualmente forma parte del río Ameca.

Clima: En el ANPPB se registra el tipo $\mathrm{C}(\mathrm{w})(\mathrm{w})[\mathrm{C}=$ clima templado subhúmedo, $(\mathrm{w})=$ lluvias en verano, $(\mathrm{w})=$ precipitación invernal del 5\% total anual] (INEGI, 2017). De acuerdo con la estación meteorológica 14269 Ahualulco de Mercado, la temperatura media anual es de $21.0^{\circ} \mathrm{C}$ con máxima y mínima de $11.7^{\circ} \mathrm{C}$ y $30.3^{\circ} \mathrm{C}$ respectivamente. La precipitación promedio anual es de $853 \mathrm{~mm}$ (CONAGUA, 2017). Hay dos periodos muy bien definidos: el de lluvias y el de estiaje. Como diagrama general, las lluvias se presentan de julio a septiembre, aunque algunas veces en mayo, junio y octubre. La temporada de estiaje inicia en octubre y se extiende hasta junio. Este último periodo puede dividirse en frío y cálido. Los meses más fríos son octubre, noviembre, diciembre y enero. Por el contrario, los más cálidos son febrero, marzo, abril y mayo.

Vegetación: El ANPPB se encuentra sobre la Sierra del Águila. Esta formación montañosa tiene un gradiente de elevación que va de los 1416 a los 2590 m s.n.m. Entre los 2590 y los 1800 m s.n.m. se desarrolla el bosque mixto de Quercus y Pinus. Las especies dominantes en estos tipos de bosques son Quercus resinosa, Q. magnoliifolia, Pinus oocarpa, P. devoniana y P. lumholtzii. Entre los 1900 y los 1500 m s.n.m. se desarrolla el bosque de Quercus. En las partes bajas, entre 1700 y los $1400 \mathrm{~m}$ s.n.m., se registral al bosque tropical caducifolio. Otros tipos de vegetación reportados en la zona son el bosque de galería, la vegetación acuática, el matorral espinoso y el pastizal inducido, así como área de cultivo de Agave tequilana y Zea mays (Gobierno de Jalisco, 2010).

\section{Trabajo de campo}

El trabajo de campo consistió en 12 excursiones botánicas realizadas entre 2011 y 2016. Durante este periodo se acumularon 24 exploraciones y se colectaron 580 muestras, de las que se elaboraron el mismo número de ejemplares botánicos de acuerdo a los protocolos descritos por Judd et al. (2002) y Lot y Chiang (1986).

Las muestras recolectadas durante el trabajo de campo fueron procesadas en el Instituto de Botánica de la Universidad de Guadalajara. El proceso implicó el secado, identificación, etiquetado, montado, registro y congelado de acuerdo con Germán (1986). Un juego completo de la colección fue depositado en el Herbario Luz María Villarreal de Puga IBUG. Los duplicados fueron distribuidos a los herbarios MEXU, IEB, ENCB y ZEA (acrónimos de acuerdo con Thiers, 2017).

\section{Trabajo de gabinete}

La determinación de los especímenes se realizó mediante la consulta de diferentes fuentes (Cervantes-Aceves, 1992; Espejo-Serna y López-Ferrari, 2001; González-Villarreal, 1986, 1990; Harker, 2002; McVaugh, 1992, 1983, 1984, 1985, 1987, 1989; Morales, 1998; Rzedowski et al., 2005) incluyendo glosarios especializados (Font-Quer, 2000; Moreno, 1984; Sousa y Zárate, 1988). Los especímenes identificados se cotejaron con ejemplares existentes en la colección del herbario IBUG. Algunos ejemplares fueron determinados por especialistas. Finalmente, la clasificación de los tipos de vegetación sigue la propuesta de Rzedowski (2006).

A partir del trabajo de campo y consulta de ejemplares de herbario, se generó una base de datos con todos los registros obtenidos. La información contenida en la base de datos se agrupó en cuatro categorías: 1) datos taxonómicos, incluidos familia, género, especie, autor; 2) datos geográficos como localidad, altitud, latitud y longitud; 3) ecológicos tales como hábitat, observaciones y fecha de colecta; 4) curatoriales como nombre del colector y número de colecta, determinador y colección. La base de datos fue utilizada para la generación del listado florístico y las etiquetas de los ejemplares. 
Se analizó la distribución geográfica de las especies siguiendo a González-Gallegos et al. (2016) y Villaseñor (2016). Se cuantificó el número de especies consideradas como malezas siguiendo a Villaseñor y Espinosa-García (1998) y las especies exóticas de acuerdo con Villaseñor y Espinosa-García (2004). También se obtuvo la categoría de riesgo de las especies registradas siguiendo la NOM-059-SEMARNAT-2010 (SEMARNAT, 2010), CITES (2017) y UICN (2017).

Las familias, géneros, especies y variedades se presentan en orden alfabético y siguen los criterios del APG IV (2016). Las clases se organizaron de acuerdo con Cronquist (1981) y las divisiones siguen a Cronquist et al. (1966). Cada taxón contiene nombre completo que incluye género, especie y autor. Los autores de las especies siguen la propuesta de Tropicos (2017).

Por último, se realizó una comparación del presente trabajo con Curiel-Ballesteros (1997) y Contreras-Rodríguez et al. (2000). Ambos estudios fueron realizados en el ANPPB y cuentan con un listado florístico. También se comparó la riqueza florística del ANPPB con el Área de Protección de Flora y Fauna La Primavera (APFFLP; SEMARNAT, 2000), Área de Protección de Flora y Fauna Sierra de Quila (APFFSQ; Guerrero-Nuño, 1994) y Cerro El Tepopote (FríasCastro et al., 2013). Se seleccionaron estas áreas ya que se encuentran cercanas al ANPPB y estas presentan tipos de vegetación similares y además cuentan con un listado florístico. Para realizar la comparación se utilizó el índice de diversidad taxonómica propuesto por Squeo et al. (1998). Este se emplea para comparar áreas de diferentes tamaños con la fórmula $B=S / \operatorname{Ln} A$, donde $S$ es el número de especies registradas, $L n$ es el logaritmo natural y $A$ es el tamaño del área en $\mathrm{km}^{2}$. Para realizar una comparación más acorde, solo se cuantificaron las especies de fanerógamas reportadas.

\section{RESUlTADOS}

\section{Tipos de vegetación}

En el ANPPB predominan el bosque de Quercus y el bosque mixto de Quercus y Pinus y en menor proporción se registra el bosque tropical caducifolio y la vegetación secundaria. El bosque de Quercus cubre la mayor parte de la superficie del área de estudio y su estructura es variable. En las laderas húmedas y con suelos profundos prospera un encinar de 15 a $20 \mathrm{~m}$ de alto. El estrato arbóreo está formado por Quercus resinosa, Q. castanea y Q. gentryi. La estructura del encinar es diferente en laderas de exposición sur y suelos someros. Su estrato arbóreo está formado por individuos de 4 a $6 \mathrm{~m}$ de altura. Las especies arbóreas más comunes, en orden de frecuencia, son Quercus resinosa, Q. magnoliifolia y $Q$. castanea. Al estrato arbustivo lo integran individuos de 1 a $3 \mathrm{~m}$ de altura. Se compone principalmente por los madroños Arctostaphylos pungens y Comarostaphylis glaucescens y también podemos encontrar algunas leguminosas como Calliandra grandiflora. El estrato herbáceo es muy dinámico, las especies más comunes son: Bouvardia chrysantha, B. scabra, B. tenuifolia, Cosmos parviflorus, Crotalaria pumila, Dalea sericea, Desmodium barbatum, Euphorbia sphaerorhiza, Helianthemum glomeratum, Hexalectris brevicaulis, Loeselia ciliata, Pinguicula oblongiloba, P. parvifolia, Salvia heterotricha, Stevia ovata, Tagetes lucida, Zinnia angustifolia y Zornia thymifolia. También son frecuentes las herbáceas perennes que poseen estructuras subterráneas. Entre ellas Bessera elegans, Bletia reflexa, Calochortus purpureus, Hypoxis fibrata, Hymenocallis jaliscensis, Oxalis decaphylla, Prochnyanthes mexicana, Sisyrinchium convolutum, Sprekelia formosissima, Tigridia dugesii y T. augusta.

El bosque mixto de Quercus y Pinus prospera en las partes más altas del ANPPB. La especie dominante es Quercus resinosa y se encuentran algunos elementos dispersos de Pinus oocarpa, P. lumholtzii, P. devoniana y los madroños Arctostaphylos pungens y Comarostaphylis glaucescens. El estrato arbustivo y herbáceo es similar en el bosque de Quercus y el bosque mixto de Quercus y Pinus. Este se compone por Aeschynomene petraea, Baccharis pteronioides, Cosmos parviflorus, Coursetia pumila, Crotalaria pumila, Cuphea llavea, Dalea 
foliosa, Desmodium barbatum, Eryngium pectinatum, Hieracium pringlei, Hypericum moranense, Psacalium palmeri, Roldana sessilifolia, Stevia viscida y Telosiphonia hypoleuca. Las herbáceas perennes con estructuras subterráneas son comunes, entre ellas Bessera elegans, Echeandia occidentalis, E. pringlei, Euphorbia sphaerorhiza, Gibasis linearis, Tigridia dugesii, T. augusta, Oxalis decaphylla y con menor frecuencia se encuentran orquídeas terrestres como Bletia reflexa y Hexalectris brevicaulis.

El bosque tropical caducifolio se encuentra en laderas escarpadas con suelos bien drenados. Dentro del polígono del ANPPB, este cubre una pequeña porción en el extremo noreste. La característica principal de este tipo de vegetación es que las especies dominantes pierden sus hojas durante el periodo de estiaje. En el estrato arbóreo predominan individuos de 3 a $8 \mathrm{~m}$ de altura. Las especies más comunes son Bursera bipinnata, Ipomoea murucoides, Lysiloma acapulcense, Opuntia fuliginosa y Tecoma stans. El estrato arbustivo y herbáceo lo componen las leguminosas: Senna foetidissima, S. septemtrionalis, S. hirsuta, Vachellia farnesiana y $V$. pennatula. También proliferan Conopholis alpina, Croton ciliatoglandulifer, Helianthemum glomerulatum, Loeselia amplectens y L. ciliata.

La vegetación secundaria se desarrolla como resultado del disturbio del bosque natural. En el área de estudio, este tipo de vegetación se ha establecido por las prácticas agrícolas y pecuarias. Esta comunidad vegetal cubre la zona de recepción a visitantes. Su estructura está dada por arbustos de 2 a $4 \mathrm{~m}$ de alto y entre ellos figuran Vachellia farnesiana, V. pennatula, Opuntia fuliginosa, Ipomoea murucoides, Solanum ferrugineum, Verbesina fastigiata y V. pantoptera. En el estrato herbáceo predominan Adenophyllum cancellatum, Asclepias glaucescens, Erigeron velutipes, Eryngium heterophyllum, Galinsoga parviflora, Gomphrena nitida, Roldana kerberi, Tagetes micrantha y Vernonanthura serratuloides. Son abundantes los pastos Cynodon dactylon y Digitaria ciliaris.

\section{Diversidad florística}

A partir del trabajo de campo y consulta de ejemplares depositados en el herbario IBUG, se generó un listado de flora fanerogámica del ANPPB. El listado contiene 262 especies agrupadas en 170 géneros y 54 familias. La división Magnoliophyta fue la más diversa con 259 especies. De estas, 58 corresponden a la clase Liliopsida y 201 a Magnoliopsida (cuadro 1, apéndice I).

Cuadro 1. Diversidad florística por grupos taxonómicos encontrada en el Área Natural Protegida Piedras Bola, municipio de Ahualulco de Mercado, Jalisco, México.

\begin{tabular}{llccc}
\hline División & Clase & Familias & Géneros & Especies \\
\hline Coniferophyta & 1 & 1 & 3 \\
Magnoliophyta & Liliopsida & 11 & 42 & 58 \\
& Magnoliopsida & 42 & 127 & 201 \\
Total & & $\mathbf{5 4}$ & $\mathbf{1 7 0}$ & $\mathbf{2 6 2}$ \\
\hline
\end{tabular}

Las familias mejor representadas son Asteraceae (47 especies, 18\%), Fabaceae (40, 15\%), Poaceae $(28,11 \%)$ y Solanaceae $(15,6 \%)$. En estas familias se concentra el $50 \%$ de la flora total del área. Los géneros más diversos son Solanum (nueve especies), Quercus (ocho especies), Desmodium (cinco especies), Bouvardia, Bursera, Dalea, Ipomoea, Muhlenbergia, Phaseolus, Phoradendron y Salvia (cuatro especies cada una).

De acuerdo con el índice de diversidad taxonómica, el ANPPB presenta una riqueza florística de 279 especies por $\mathrm{km}^{2}$. En comparación con áreas cercanas, el ANPPB mostró el índice más alto (cuadro 2). Es importante tomar en cuenta el esfuerzo de muestreo entre autores, los 
criterios para delimitar las comunidades vegetales y la imprecisión en el cálculo de las superficies en las que se han realizado los muestreos (Frías-Castro et al., 2013).

Cuadro 2: Riqueza florística del ANPPB y su comparación con otras áreas cercanas utilizando el índice de diversidad taxonómica.

\begin{tabular}{|c|c|c|c|c|c|c|c|}
\hline Localidad/fuente & $\begin{array}{l}\text { Área } \\
\left(\mathrm{km}^{2}\right)\end{array}$ & Tipos de vegetación & $\begin{array}{c}\text { Número de } \\
\text { exploraciones }\end{array}$ & Familias & Géneros & Especies & $\begin{array}{c}\text { Riqueza } \\
(\mathrm{sp} / \operatorname{Ln} A)\end{array}$ \\
\hline $\begin{array}{l}\text { APFFLP } \\
\text { SEMARNAT, } 2000\end{array}$ & 305 & BTC, BPQ, BQ, VA & ND & 97 & 407 & 789 & 138 \\
\hline $\begin{array}{l}\text { APFFSQ } \\
\text { Guerrero-Nuño, } 1994\end{array}$ & 320 & $\begin{array}{l}\text { BES, BTC, BQ, BPQ, } \\
\text { BMM, BG }\end{array}$ & 45 & 116 & 404 & 740 & 128 \\
\hline $\begin{array}{l}\text { Cerro El Tepopote } \\
\text { Frías-Castro et al ., } 2013\end{array}$ & 6,55 & BQ, BPE & 30 & 66 & 195 & 339 & 197 \\
\hline $\begin{array}{l}\text { ANPPB } \\
\text { el presente trabajo }\end{array}$ & 2,56 & BQ, BQP, BTC, VS & 22 & 54 & 170 & 262 & 279 \\
\hline
\end{tabular}

NOTA: se consideran exclusivamente a las familias, géneros y especies dentro del grupo de las fanerógamas reportadas por los autores.

\section{Formas de vida}

El hábito predominante en el ANPPB es el herbáceo (175 especies, 67\%), después el arbustivo $(40,15 \%)$, arbóreo $(31,12 \%)$ y bejucos o lianas $(16,6 \%)$. El mayor número de especies se desarrollan en un hábitat terrestre $(254,97 \%)$, aunque también se encuentran especies epífitas $(7,2.6 \%)$ y rupícolas $(1,0.4 \%)$. La gran mayoría de las plantas en el ANPPB tiene un tipo de nutrición autótrofa $(254,97 \%)$ y en menor medida encontramos plantas hemiparásitas $(6$, $2.2 \%)$, holoparásitas $(1,0.4 \%)$ y saprófitas $(1,0.4 \%)$ (Apéndice I).

\section{Distribución geográfica y endemismos}

De acuerdo con la revisión, 97 (37\%) especies registradas son endémicas de México. Se registró a Echeandia pringlei, una especie endémica del estado de Jalisco y exclusiva de la Sierra del Águila y el volcán de Tequila. Se destacan once especies con una distribución restringida al occidente de México, región que incluye a los estados de Aguascalientes, Colima, sur de Durango, oeste de Guanajuato, Jalisco, oeste de Michoacán, Nayarit y sur de Zacatecas. Estas son Aldama angustifolia, Brongniartia cordata, Critoniopsis autumnalis, C. baadii, Echeandia pringlei, Electranthera cuneifolia, Hymenocallis jaliscensis, Passiflora podadenia, Polianthes longiflora, Salvia heterotricha, Vallesia spectabilis y Vernonia bealliae. Es importante resaltar que todas las especies registradas para la familia Asparagaceae y el género Salvia son endémicas de México. Por otra parte, las especies exóticas representan el 3\% (8 spp.) del total, mientras que las especies consideradas malezas ocupan el 33.5\% (88 spp.).

\section{Especies bajo protección legal}

Se registraron 15 especies bajo alguna categoría de protección. De acuerdo con la NOM-059SEMARNAT-2010 (SEMARNAT, 2010), dos especies se encuentran bajo protección legal en México. Estas son Vallesia spectabilis y Polianthes longiflora bajo la categoría de protección especial (Pr, endémica). En el apéndice II de CITES (2017) se enlistan tres especies registradas en el ANPPB: Opuntia fuliginosa, Euphorbia strigosa y Hexalectris brevicaulis. Por último, de acuerdo con la UICN (2017) se registran 10 especies bajo alguna categoría de protección. Nueve de ellas (Arbutus xalapensis, Calochortus purpureus, Eysenhardtia polystachya, Lysiloma acapulcense, Opuntia fuliginosa, Pinus devoniana, P. oocarpa, Quercus castanea, Senna foetidissima) se encuentran bajo la categoría de preocupación menor (LC) y solo Pinus lumholtzii como casi amenazada (NT). 


\section{DISCUSIÓN}

De acuerdo con Rzedowski (2006), el bosque de Quercus, en conjunto con el bosque de Pinus, constituyen los tipos de vegetación dominantes en las áreas con clima templado y semihúmedo. De igual forma, los bosques mixtos de Quercus y Pinus son muy frecuentes en las zonas montañosas del país. Los tipos de vegetación dominantes en el ANPPB son el bosque de Quercus y el bosque mixto de Quercus y Pinus. Las condiciones climáticas, topográficas, geológicas y edafológicas presentes en el ANPPB favorecen la dominancia de estos tipos de vegetación. Por su parte, el bosque tropical caducifolio también es un elemento común en la zona, específicamente en las partes bajas de la Sierra del Águila. En general, el área se encuentra conservada a pesar de la ganadería presente dentro del polígono del área natural.

Las familias mejor representadas en el ANPPB fueron Asteraceae, Fabaceae, Poaceae y Solanaceae. De acuerdo con el listado de plantas vasculares nativas de México presentado por Villaseñor (2016), Asteraceae y Fabaceae cuentan con el mismo patrón de diversidad que en el ANPPB. Poaceae se encuentra como la cuarta familia más diversa, por debajo de Orchidaceae. Por último, Solanaceae se encuentra dentro de las primeras 15 familias más diversas.

A pesar de su reducido tamaño, el ANPPB ofrece novedades botánicas. Se registra una nueva localidad para Vallesia spectabilis (Apocynaceae) que se conocía solo de las sierras de San Sebastián del Oeste y Manantlán. Se trata de una especie sumamente rara, con poblaciones muy reducidas, por lo que su probabilidad de extinción es alta (Cuevas-Guzmán et al., 2004; Morales, 1998). Por esta razón, Vallesia spectabilis está sujeta a protección especial de acuerdo con la Norma Oficial Mexicana NOM-059-SEMARNAT-2010 (SEMARNAT, 2010). De igual forma, se colectó a Lycianthes pringlei (Solanaceae), un arbusto endémico de México, el cual solo crece en la parte Centro-Occidente de la Faja Volcánica Transmexicana. Esta es una especie raramente colectada (Dean et al., 2007). Se registra una nueva localidad para Echeandia pringlei (Anthericaceae) con localidad tipo en Etzatlán. Por último, se registró a Polianthes longiflora (Agavaceae), una especie sujeta a protección especial por su grado de endemismo de acuerdo con la Norma Oficial Mexicana NOM-059-SEMARNAT-2010 (SEMARNAT, 2010).

Al realizar una comparación con estudios previos dentro del ANPPB destaca la clasificación de los tipos de vegetación. En los trabajos de Curiel-Ballesteros (1997) y Contreras-Rodríguez et al. (2000) se describen cuatro tipos de vegetación de acuerdo con Miranda y Hernández (1963): bosque latifoliado esclerófilo caducifolio, bosque de galería, matorral y selva baja caducifolia. En el presente estudio, de acuerdo con Rzedowski (2006), se describen cuatro tipos de vegetación: bosque de Quercus, bosque mixto de Quercus y Pinus, bosque tropical caducifolio y vegetación secundaria. Al realizar una homologación de las nomenclaturas resalta el hecho de que en el presente estudio no se incluye el bosque de galería como un tipo de vegetación presente. La comunidad asociada a cañadas húmedas se describe como una variante del bosque de Quercus. Se clasificó de esta forma porque el escurrimiento no es permanente, ni más o menos permanente como denota Rzedowski (2006) en su descripción del bosque de galería. También, el estrato arbóreo está dominado por especies del género Quercus y no se registraron especies con afinidades riparias.

Se observaron diferencias al comparar los listados florísticos de Curiel-Ballesteros (1997), Contreras-Rodríguez et al. (2000) y el presente estudio. Curiel-Ballesteros (1997) presenta un listado florístico que incluye 125 especies, 90 géneros y 36 familias. Por su parte, ContrerasRodríguez et al. (2000) reportan 139 especies, 99 géneros y 43 familias. En el presente trabajo se enlista un mayor número de taxa, en total se reportan 262 especies, 170 géneros y 54 familias. Es importante resaltar que los tres estudios comparados tienen objetivos diferentes. Curiel-Ballesteros (1997) realizó una descripción general del medio abiótico en donde menciona las principales especies de flora y fauna que se pueden encontrar en la zona. Contreras-Rodríguez et al. (2000) realizaron un estudio ecológico. En él, se realizaron transectos de franja, con el objetivo de determinar la frecuencia, densidad, dominancia e índice de importancia para las especies muestreadas en Piedras Bola. El presente estudio está enfocado 
a la descripción de la flora y vegetación del área. El inventario aquí presentado es el resultado de un muestreo completamente al azar. El tamaño del polígono del ANPPB es relativamente pequeño, lo que dio la oportunidad de realizar recolectas botánicas en toda su extensión. Por consiguiente, el esfuerzo de muestreo fue mayor en el presente trabajo en comparación con estudios previos realizados en el ANPPB.

Por último, no fue posible comparar la riqueza florística entre Curiel-Ballesteros (1997), Contreras-Rodríguez et al. (2000) y el presente estudio utilizando el índice de diversidad taxonómica. La razón se fundamenta en que los trabajos referidos presentan inconsistencias en cuanto a la superficie muestreada. La clara delimitación del área de estudio es clave para realizar la comparación con el índice.

La comparación con el índice de diversidad taxonómica muestra las siguientes observaciones. El APFFLP y el APFFSQ resultaron con índices similares (138 y 128 especies por $\mathrm{km}^{2}$, respectivamente), también estas dos áreas tienen una superficie afín (305 y $320 \mathrm{~km}^{2}$ ) (Cuadro 2). En comparación con el tamaño del ANPPB $\left(2.56 \mathrm{~km}^{2}\right)$, la diferencia es significativa. A pesar de que el índice de diversidad taxonómica se utiliza para comparar áreas de distintas dimensiones, en este caso en particular las dimensiones son muy diferentes. El Cerro El Tepopote cuenta con la superficie más similar a la del ANPPB que las otras áreas comparadas (6.55 y $2.56 \mathrm{~km}^{2}$, respectivamente). Al comparar el índice de diversidad taxonómica entre estos sitios, el ANPPB resultó con una cifra mayor. Aunque el ANPPB presenta un índice mayor y más tipos de vegetación, en el Cerro El Tepopote se registró un mayor número de especies fanerógamas (262 y 339, respectivamente) y además se describió a Dahlia pugana como producto del inventario (Castro-Castro et al., 2012). Lo anterior muestra que el Cerro El Tepopote es un área bien conservada. Se estima que la dificultad del terreno y el difícil acceso el Cerro El Tepopote son los factores que favorecen su conservación. En el Cerro El Tepopote no es común la ganadería, mientras que sí se ha observado en el ANPPB. Por último, el número de visitantes es muy elevado en el ANPPB en comparación con el Cerro El Tepopote. En conclusión, es importante resaltar que el ANPPB cuenta con una superficie más pequeña y menos escarpada que las áreas comparadas. Estos factores favorecieron la intensidad del muestreo, lo que permitió una mejor representación de la diversidad florística. Se estima que el esfuerzo de muestreo del presente estudio es alto.

\section{Conclusiones}

La información generada en el presente estudio contribuye al conocimiento florístico de la Sierra del Águila y en particular a la del ANPPB. En el área de Piedras Bola son evidentes varios factores de perturbación. Entre ellos destacan la ganadería extensiva, el senderismo, la generación de residuos sólidos urbanos y el vandalismo. Se recomienda dar seguimiento al programa de conservación y manejo propuesto por Castillo-Girón y Aceves-Ávila (2007) así como aplicar el reglamento oficial del ANPPB (SEGOB, 2012). La alta riqueza de especies en comparación con áreas cercanas y la presencia de las megaesferulitas, son indicadores de la importancia de la conservación del área. Con la presente aportación se espera difundir la importancia florística del ANPPB y promover su protección y conservación.

\section{AGRADECIMIENTOS}

Al H. Ayuntamiento Constitucional administración 2010-2012 del municipio de Ahualulco de Mercado y a los pobladores de Santa Cruz de Bárcenas por el apoyo en la realización del trabajo de campo. A Rodríguez-Zaragoza, F. y Rosas-Espinoza, V. por la organización del proyecto. Al Instituto de Botánica de la Universidad de Guadalajara por las facilidades proporcionadas para el procesamiento de las muestras. A los siguientes especialistas por la determinación de materia: Carrillo-Reyes, P. (Crassulaceae); Castro-Castro, A. (Apocynaceae, 
Asteraceae); González-Castro, S. (Poaceae); González-Gallegos, J. G. (Lamiaceae); GonzálezVillareal, L. M. (Fagaceae, Ericaceae); Harker, M. (Asteraceae); Pérez de la Rosa, J. A. (Pinaceae), Reynoso-Dueñas, J. J. (Fabaceae, Poaceae); Rodríguez-Contreras, A. (Iridaceae, Asparagaceae, Amaryllidaceae, Solanaceae) y Thomas, D. (Acanthaceae). Fue importante el apoyo en campo de los colegas Sánchez-Chávez, E., Anguiano-Constante, M. y VázquezBolaños, M. A Anguiano-Constante, M. y Ramírez-Díaz, C. por la revisión y recomendaciones al presente trabajo. Agradecemos las sugerencias del editor Fernández-Nava, R. y de los tres revisores anónimos que ayudaron a mejorar el manuscrito.

\section{LiTERATURA CITADA}

APG IV. (2016). An update of the Angiosperm Phylogeny Group classification for the orders and families of flowering plants: APG IV. Botanical Journal of the Linnean Society, 181(1), 1-20. https://doi.org/10.1111/boj.12385

Castillo-Girón, V. M., y Aceves-Ávila, C. (2007). Gestión para la sustentabilidad del Área Natural Piedras Bola, Ahualulco de Mercado, Jalisco. Guadalajara, Mexico: Universidad de Guadalajara.

Castro-Castro, A., Rodríguez, A., Vargas-Amado, G., y Harker, M. (2012). Diversidad del género Dahlia (Asteraceae: Coreopsideae) en Jalisco, México y descripción de una especie nueva. Revista Mexicana de Biodiversidad, 83(2), 347-358. https://doi.org/10.7550/rmb.25082

Cervantes-Aceves, N. (1992). La familia Malvaceae en el estado de Jalisco, México (Colección Flora de Jalisco 3). Guadalajara, Mexico: Instituto de Botánica, Universidad de Guadalajara.

CITES. (2017). Lista de especies CITES. Recuperado el 18 de enero de 2017, a partir de http://checklist.cites.org/\#/es

CONAGUA. (2017). Normales climatológicas de Ahualulco de Mercado. Recuperado el 13 de mayo de 2017, a partir de http://smn.cna.gob.mx/

Contreras-Rodríguez, S. H., Romo-Campos, R., y Reynoso-Dueñas, J. J. (2000). Caracterización de la vegetación en la zona de Piedras Bola, Ahualulco de Mercado, Jalisco, México. Boletín del Instituto de Botánica, 7, 103-121.

Cronquist, A. (1981). An integrated system of classification of flowering plants. Nueva York, EUA: Columbia University Press.

Cronquist, A., Takhtajan, A., y Zimmermann, W. (1966). On the higher taxa of Embryobionta. Taxon, 15(4), 129-134.

Cuevas-Guzmán, R., Núñez-López, N. M., Sánchez-Rodríguez, E. V., y Solís-Magallanes, A. (2004). Especies nuevas y descritas de plantas de la Estación Científica Las Joyas y áreas aledañas. En R. E. Cuevas-Guzmán y J. Jardel-Peláez (Eds.), Flora y vegetación de la Estación Científica Las Joyas (pp. 232-262). Guadalajara, Mexico: Universidad de Guadalajara.

Curiel-Ballesteros, A. (1997). Propuesta para declarar Área Natural Protegida la Zona de Piedras Bola Municipio de Ahualulco de Mercado, Jalisco, México. Guadalajara, Mexico: Universidad de Guadalajara.

Dean, E. A., Walden, G. K., y Thrasher, S. L. (2007). Lycianthes pringlei (Solanaceae), a rarely collected shrub of western Mexico. Brittonia, 59(1), 49-53.

Espejo-Serna, A., y López-Ferrari, A. R. (2001). Flora de Veracruz (Hypoxidaceae) Fasículo 120. México: Instituto de Ecología, A.C.

Espinosa, D., y Ocegueda, S. (2007). Introducción. En I. Luna, J. J. Morrone, y D. Espinosa (Eds.), Biodiversidad de la Faja Volcánica Transmexicana (pp. 5-6). México, D.F.: Universidad Autónoma de México.

Font-Quer, P. (2000). Diccionario de Botanica. Barcelona, España: Ediciones Península.

Frías-Castro, A., Castro-Castro, A., González-Gallegos, J. G., Suárez-Muro, E. A., y RendónSandoval, F. J. (2013). Flora vascular y vegetación del Cerro El Tepopote, Jalisco, México. Botanical Sciences, 91(1), 53-74. https://doi.org/10.17129/botsci.402 
Germán, M. T. (1986). Estructura y organización del herbario. En A. Lot y F. Chiang (Eds.), Manual de herbario: administración y manejo de colecciones, técnicas de recolección y preparación de ejemplares botánicos (pp. 11-30). México, D.F.: Consejo Nacional de Flora de México A.C.

Gobierno de Jalisco. (2007). Declaratoria del Área Natural Protegida bajo categoría de manejo de formaciones naturales de interés municipal la zona conocida como "Piedras Bola", con una superficie de 256 hectáreas, ubicadas en el municipio de Ahualulco de Mercado, Jalisco. Periódico Oficial 14 de febrero del 2007 número 28, sección VIII, tomo CCCLVI).

Gobierno de Jalisco. (2010). Declaratoria de Área Natural Protegida de competencia estatal relativa al Área Estatal de Protección Hidrológica "Sierra del Águila" con una superficie de 20,746.3727 hectáreas, ubicadas en los municipios de Etzatlán, Ameca, Ahualulco de Mercado y San Juanito de Escobedo, todos del Estado de Jalisco. Periódico Oficial 16 de febrero del 2010 número 47, sección II, tomo CCCLXV.

González-Gallegos, J. G., Castro-Castro, A., Quintero-Fuentes, V., Mendoza-López, M. E., y De Castro-Arce, E. (2016). Revisión taxonómica de Lamiceae del occidente de México. Ibugana, 7(1), 1-545.

González-Villarreal, L. M. (1986). Contribución al conocimiento del género Quercus en el estado de Jalisco, México (Colección Flora de Jalisco 1). Guadalajara, Mexico: Instituto de Botánica, Universidad de Guadalajara.

González-Villarreal, L. M. (1990). Las Ericáceas de Jalisco, México (Colección Flora de Jalisco 2). Guadalajara, Mexico: Instituto de Botánica, Universidad de Guadalajara.

Guerrero-Nuño, J. J. (1994). Contribución al conocimiento de la vegetación y la flora de la Sierra de Quila, Jalisco, México. Centro Universitario de Ciencias Biológicas y Agropecuarias. Universidad de Guadalajara.

Harker, M. (2002). La familia Compositae (Asteraceae) en la Barranca del Río Santiago, Municipio de Guadalajara, Jalisco, México: Un modelo para la conservación. Centro Universitario de Ciencias Biológicas y Agropecuarias. Universidad de Guadalajara.

INEGI. (2017). Mapa digital de México. Recuperado el 2 de mayo de 2017, a partir de http://www.inegi.org.mx/geo/contenidos/mapadigital/

Judd, W. S., Campbell, C. S., Kellogg, E. A., Stevens, P. F., y Donoghue, M. J. (2002). Plant Systematics, a Phylogenetic Approach (Segunda ed). Massachusetts, EUA: Sinauer Associates, Inc.

Lot, A., y Chiang, F. (1986). Manual de herbario: Administración y manejo de colecciones, técnicas y preparación de ejemplares botánicos. México, D.F.: Consejo Nacional de Flora de México A.C.

Lugo-Hubp, J. (2004). La Ciencia Para Todos 201: El relieve de la tierra y otras sorpresas. México, D.F.: Fondo de Cultura Económica.

McVaugh, R. (1983). Flora Novo-Galiciana XIV (Gramineae). Ann Arbor, EUA: University of Michigan Press.

McVaugh, R. (1984). Flora Novo-Galiciana XII (Compositae). Ann Arbor, EUA: University of Michigan Press.

McVaugh, R. (1985). Flora Novo-Galiciana XVI (Orchidaceae). Ann Arbor, EUA: University of Michigan Press.

McVaugh, R. (1987). Flora Novo-Galiciana V(Leguminosae). Ann Arbor, EUA: University of Michigan Press.

McVaugh, R. (1989). Flora Novo-Galiciana XV (Bromeliaceae to Dioscoreaceae). Ann Arbor, EUA: University of Michigan Press.

McVaugh, R. (1992). Flora Novo-Galiciana XVII (Gymnospems to Pteridophytes). Ann Arbor, EUA: University of Michigan Press.

Miranda, F., y Hernandez, E. (1963). Los tipos de vegetación de México y su clasificación. Boletín de la Sociedad Botánica de México, 28, 29-179. https://doi.org/10.17129/botsci.1084

Morales, J. F. (1998). Three new species and a new combination in Vallesia (Apocynaceae). Novon, 8(3), 263-264. https://doi.org/10.2307/3392016 
Recibido:

19/julio/2017

Aceptado:

12/marzo/2018
Moreno, N. (1984). Glosario Botánico Ilustrado. México, D.F.: CECSA.

Rzedowski, J. (2006). Vegetación de México. México, D.F.: Comisión Nacional para el Conocimiento y Uso de la Biodiversidad.

Rzedowski, G. C. de, Rzedowski, J., y Colaboradores. (2005). Flora fanerogámica del Valle de México. Pátzcuaro, Michoacán: Instituto de Ecología, A.C. y Comisión Nacional para el Conocimiento y Uso de la Biodiversidad.

SEGOB. (2012). Reglamento de Piedras Bola (Orden Jurídico Nacional). Recuperado el 17 de septiembre de 2016, a partir de http://www.ordenjuridico.gob.mx/despliegaedo3. php?edo=14\&orderSelectionado=523\&catTipo=523

SEMARNAT. (2000). Programa de Manejo Área de Protección de Flora y Fauna La Primavera. México, D.F.: SEMARNAT.

SEMARNAT. (2010). Norma Oficial Mexicana NOM-059-SEMARNAT-2010: Protección Ambiental-Especies nativas de México de flora y fauna silvestres-Categorías de riesgo y especificaciones para su inclusión, exclusión o cambio-Lista de especies en riesgo. México, D.F.: Diario Oficial de la Federación 30 de diciembre de 2010; Segunda sección.

SEMARNAT. (2013). Informe de la situación del medio ambiente. Compendio de estadísticas ambientales. Indicadores clave $y$ de desempeño ambiental. México, D.F.: SEMARNAT.

Sousa, S. M., y Zárate, S. (1988). Flora Mesoamericana: glosario para Spermatophyta, español-inglés. México, D.F.: Universidad Nacional Autónoma de México.

Squeo, F. A., Cavieres, L. A., Arancio, G., Novoa, J. E., Matthei, O., Marticorena, C., ... Muñoz, M. (1998). Biodiversidad de la flora vascular en la Región de Antofagasta, Chile. Revista Chilena de Historia Natural, 71, 571-591.

Stirling, M. (1969). Solving the mystery of Mexico's Great Stone Spheres. National Geographic, 136(2), 294-300.

Thiers, B. (2017). Index Herbariorum: A global directory of public herbaria and associated staff. New York Botanical Garden's Virtual Herbarium. Recuperado a partir de http://sweetgum.nybg.org/science/ih/

Tropicos. (2017). Missouri Botanical Garden. Recuperado a partir de http://www.tropicos.org

UICN. (2017). Lista Roja de Especies. Recuperado a partir de http://www.iucnredlist.org/

Villaseñor, J. L. (2016). Catálogo de las plantas vasculares nativas de México. Revista Mexicana de Biodiversidad, 87(3), 559-902. https://doi.org/10.1016/j.rmb. 2016.06.017

Villaseñor, J. L., y Espinosa-García, F. J. (1998). Catálogo de malezas de México. México, D.F.: Universidad Nacional Autónoma de México. Consejo Nacional Consultivo Fitosanitario y Fondo de Cultura Económica.

Villaseñor, J. L., y Espinosa-García, F. J. (2004). The alien flowering plants of Mexico. Diversity and Distributions, 10(2), 113-123. https://doi.org/10.1111/j.13669516.2004.00059.x 
Apéndice 1. Listado florístico del Área Natural Protegida Piedras Bola.

Colectores (Cols.): AN (A. Navarro), ANA (Alicia Nuño-Amézquita), ARC (Aarón Rodríguez-Contreras), DJ (D. Juárez), EHM (E. Hernández-M.), ERR (E. Reyes-R.), ESC (Erandi Sánchez-Chávez), FRZ (F. Rodríguez-Zaragoza), FVU (Fernando VázquezUribe), JJRD (Jesús Jacqueline Reynoso-Dueñas), JL (J. Leal), LMGV (Luz María González-Villarreal), LMTP (L.M. Tapia-P.), LMVP (Luz María Villarreal de Puga), MAGM (Miguel Ángel García-Martínez), MAC (Marco Anguiano-Constante), MCB (M. Cházaro-Basañes), MVB (Miguel Vázquez-Bolaños), RMcV (Rogers McVaugh), SP (S. Pérez). Forma biológica. A (hábito): a (árbol), r (arbusto), h (hierba), b (bejuco o liana). H (hábitat): E (epífito), R (rupícola), T (terrestre). N (nutrición): A (autótrofa), M (hemiparásita), L (holoparásita), S (saprófita). Símbolos: especie endémica de México $(*)$.

\begin{tabular}{|c|c|c|c|}
\hline $\mathbf{A}$ & Familia/Especie & Cols./números de colecta & $\mathbf{H} / \mathbf{N}$ \\
\hline & DIVISIÓN PINOPHYTA & & \\
\hline & Pinaceae & & \\
\hline $\mathbf{a}$ & Pinus devoniana Lindl. & MAGM 133 & T/A \\
\hline $\mathbf{a}$ & *Pinus lumholtzii B.L. Rob. \& Fernald & MAGM \& ESC 46 & $\mathrm{~T} / \mathrm{A}$ \\
\hline \multirow[t]{4}{*}{$\mathbf{a}$} & Pinus oocarpa Schiede ex Schltdl. & MAGM \& ESC 50 & $\mathrm{~T} / \mathrm{A}$ \\
\hline & DIVISIÓN MAGNOLIOPHYTA & & \\
\hline & CLASE LILIOPSIDA & & \\
\hline & Asparagaceae & & \\
\hline $\mathbf{h}$ & *Bessera elegans Schult. f. & MAGM 115; MAGM 63 & T/A \\
\hline $\mathbf{h}$ & *Echeandia durangensis (Greenm.) Cruden & MAGM \& ESC 44 & $\mathrm{~T} / \mathrm{A}$ \\
\hline $\mathbf{h}$ & *Echeandia occidentalis Cruden & MAGM \& ESC 52 & $\mathrm{~T} / \mathrm{A}$ \\
\hline $\mathbf{h}$ & *Echeandia pringlei Greenm. & MAGM et al. 123 & T/A \\
\hline $\mathbf{h}$ & *Manfreda jaliscana Rose & MAGM \& ARC 164 & T/A \\
\hline $\mathbf{h}$ & *Polianthes longiflora Rose & MAGM \& ESC 58 & T/A \\
\hline h & *Prochnyanthes mexicana (Zucc.) Rose & MAGM et al. 126 & T/A \\
\hline h & *Hymenocallis jaliscensis M.E. Jones & MAGM \& DJ 247 & T/A \\
\hline \multirow[t]{2}{*}{ h } & *Sprekelia formosissima (L.) Herb. & MAGM \& ARC 236 & $\mathrm{~T} / \mathrm{A}$ \\
\hline & Bromeliaceae & & \\
\hline $\mathbf{h}$ & *Tillandsia achyrostachys E. Morrenn ex Baker & MAGM 205 & $\mathrm{E} / \mathrm{A}$ \\
\hline $\mathbf{h}$ & Tillandsia recurvata $(\mathrm{L}.) \mathrm{L}$. & MAGM 203 & $\mathrm{E} / \mathrm{A}$ \\
\hline \multirow[t]{2}{*}{$\mathbf{h}$} & *Viridantha plumosa (Baker) Espejo & MAGM 194, 206 & E/A \\
\hline & Commelinaceae & & \\
\hline $\mathbf{h}$ & Commelina erecta $\mathrm{L}$. & MAGM \& ESC 47 & T/A \\
\hline $\mathbf{h}$ & Commelina tuberosa $\mathrm{L}$. & JJRD 2943 & T/A \\
\hline $\mathbf{h}$ & *Gibasis linearis (Benth.) Rohweder & ARC et al. 6194; MAGM 9 & $\mathrm{~T} / \mathrm{A}$ \\
\hline $\mathbf{h}$ & Tinantia longipedunculata Standl. \& Steyerm. & MAGM \& ANA 93 & T/A \\
\hline \multirow[t]{2}{*}{$\mathbf{h}$} & *Tinantia macrophylla S. Watson & MAGM \& ANA 94 & T/A \\
\hline & Cyperaceae & & \\
\hline \multirow[t]{2}{*}{$\mathbf{h}$} & Cyperus aggregatus (Willd.) Endl. & FVU 10 & T/A \\
\hline & Dioscoreaceae & & \\
\hline \multirow[t]{2}{*}{$\mathbf{b}$} & *Dioscorea militaris B.L. Rob. & MAGM \& ANA 92 & $\mathrm{~T} / \mathrm{A}$ \\
\hline & Hypoxidaceae & & \\
\hline $\mathbf{h}$ & Hypoxis fibrata Brackett & ARC et al. 6195; MAGM 226 & T/A \\
\hline $\mathbf{h}$ & $\begin{array}{l}\text { Hypoxis mexicana Schult. \& Schult. f. } \\
\text { Iridaceae }\end{array}$ & MAGM \& ARC 226 & T/A \\
\hline $\mathbf{h}$ & Sisyrinchium convolutum Nocca & MAGM \& ARC 227 & T/A \\
\hline $\mathbf{h}$ & *Tigridia augusta Drapiez & ARC et al. 6187; MAGM 243 & $\mathrm{~T} / \mathrm{A}$ \\
\hline $\mathbf{h}$ & *Tigridia dugesii S. Watson & ARC et al. 6186; MAGM 231 & $\mathrm{~T} / \mathrm{A}$ \\
\hline \multirow[t]{2}{*}{$\mathbf{h}$} & *Calochortus purpureus (Kunth) Baker & MAGM \& ANA 62,73 & T/A \\
\hline & Orchidaceae & & \\
\hline $\mathbf{h}$ & Bletia reflexa Lindl. & MAGM 144 & T/A \\
\hline $\mathbf{h}$ & Dichromanthus aurantiacus (La Llave \& Lex.) Salazar \& Soto Arenas & MAGM \& ESC 19, 27, 57 & T/A \\
\hline $\mathbf{h}$ & Habenaria clypeata Lindl. & MAGM \& ESC 33 & $\mathrm{~T} / \mathrm{A}$ \\
\hline $\mathbf{h}$ & *Hexalectris brevicaulis L.O. Williams & MAGM \& ESC 1 & $\mathrm{~T} / \mathrm{S}$ \\
\hline
\end{tabular}




\begin{tabular}{|c|c|c|c|}
\hline \multirow{2}{*}{$\frac{\mathbf{A}}{\mathbf{h}}$} & Familia/Especie & Cols./números de colecta & $\mathbf{H} / \mathbf{N}$ \\
\hline & $\begin{array}{l}\text { Spiranthes graminea Lindl. } \\
\text { Poaceae }\end{array}$ & MAGM \& ARC 229 & T/A \\
\hline h & Aegopogon cenchroides Humb. \& Bonpl. ex Willd. & MAGM \& MAC 303 & T/A \\
\hline h & Aristida laxa Cav. & SP \& AN 371 & $\mathrm{~T} / \mathrm{A}$ \\
\hline h & Bouteloua curtipendula (Michx.) Torr. & MAGM \& MAC 299, 315 & $\mathrm{~T} / \mathrm{A}$ \\
\hline $\mathbf{h}$ & Bouteloua repens (Kunth) Scribn. \& Merr. & MAGM \& MAC 304 & $\mathrm{~T} / \mathrm{A}$ \\
\hline h & Chloris radiata (L.) Sw. & MAGM \& MAC 308 & $\mathrm{~T} / \mathrm{A}$ \\
\hline $\mathbf{h}$ & Cynodon dactylon (L.) Pers. & MAGM \& DJ 245 & T/A \\
\hline h & Digitaria ciliaris (Retz.) Koeler & MAGM \& MAC 299, 315 & T/A \\
\hline h & Digitaria ternata (Hochst. ex A. Rich.) Stapf & MAGM \& MAC 292, 301 & $\mathrm{~T} / \mathrm{A}$ \\
\hline h & Eleusine indica (L.) Gaertn. & MAGM \& MAC 311 & $\mathrm{~T} / \mathrm{A}$ \\
\hline h & Eleusine tristachya (Lam.) Lam. & MAGM \& MAC 291 & $\mathrm{~T} / \mathrm{A}$ \\
\hline h & Eragrostis mexicana (Hornem.) Link & MAGM \& MAC 300 & T/A \\
\hline h & *Eragrostis plumbea Scribn. ex Beal & MAGM \& MAC 312 & $\mathrm{~T} / \mathrm{A}$ \\
\hline h & Festuca arundinacea Schreb. & MAGM \& MAC 320 & T/A \\
\hline h & *Hilaria ciliata (Scribn.) Nash & MAGM 134 & $\mathrm{~T} / \mathrm{A}$ \\
\hline h & Muhlenbergia ciliata (Kunth) Trin. & JJRD 2967 & $\mathrm{~T} / \mathrm{A}$ \\
\hline h & Muhlenbergia pereilema P.M. Peterson & MAGM \& MAC 305,310 & $\mathrm{~T} / \mathrm{A}$ \\
\hline h & Muhlenbergia robusta (E. Fourn.) Hitchc. & MAGM \& MAC 309 & $\mathrm{~T} / \mathrm{A}$ \\
\hline h & *Muhlenbergia uniseta (Lag.) Columbus & MAGM \& MAC 294, 306 & $\mathrm{~T} / \mathrm{A}$ \\
\hline h & Oplismenus burmannii (Retz.) P. Beauv. & MAGM \& MAC 298 & T/A \\
\hline $\mathbf{r}$ & *Otatea acuminata (Munro) C.E. Calderón \& Soderstr. & MAGM 132 & $\mathrm{~T} / \mathrm{A}$ \\
\hline h & Panicum hirticaule J. Presl & MAGM \& MAC 322 & $\mathrm{~T} / \mathrm{A}$ \\
\hline h & Paspalum convexum Humb. \& Bonpl. ex Flüggé & MAGM \& MAC 295, 297, 302 & $\mathrm{~T} / \mathrm{A}$ \\
\hline h & Paspalum notatum Flüggé & MAGM \& MAC 296, 319 & T/A \\
\hline h & Paspalum paniculatum $\mathrm{L}$. & MAGM \& MAC 323 & $\mathrm{~T} / \mathrm{A}$ \\
\hline h & Rhynchelytrum repens (Willd.) C.E. Hubb. & MAGM \& MAC 293,316, 324 & T/A \\
\hline h & Setaria parviflora (Poir.) Kerguélen & MAGM \& MAC 326 & $\mathrm{~T} / \mathrm{A}$ \\
\hline h & Sporobolus indicus (L.) R. Br. & MAGM \& MAC 313,321 & $\mathrm{~T} / \mathrm{A}$ \\
\hline $\mathbf{h}$ & Stenotaphrum secundatum (Walter) Kuntze & MAGM \& MAC 317 & $\mathrm{~T} / \mathrm{A}$ \\
\hline & CLASE MAGNOLIOPSIDA & & \\
\hline & Acanthaceae & & \\
\hline h & *Dyschoriste xylopoda Kobuski & MAGM \& ESC 22 & T/A \\
\hline h & *Henrya scorpioides (L.) Ness & JJRD 2942 & T/A \\
\hline $\mathbf{h}$ & $\begin{array}{l}\text { Pseuderanthemum praecox (Benth.) Leonard } \\
\text { Amaranthaceae }\end{array}$ & MAGM 189 & $\mathrm{~T} / \mathrm{A}$ \\
\hline h & Amaranthus hybridus L. & MAGM 127 & $\mathrm{~T} / \mathrm{A}$ \\
\hline h & Gomphrena nitida Rothr. & MAGM 122 & $\mathrm{~T} / \mathrm{A}$ \\
\hline h & Gomphrena serrata $\mathrm{L}$. & MAGM \& ANA 88 & T/A \\
\hline h & $\begin{array}{l}\text { Iresine diffusa Humb. \& Bonpl. ex Willd. } \\
\text { Apiaceae }\end{array}$ & MAGM 135 & $\mathrm{~T} / \mathrm{A}$ \\
\hline h & Eryngium heterophyllum Engelm. & MAGM 151 & T/A \\
\hline $\mathbf{h}$ & $\begin{array}{l}\text { *Eryngium pectinatum C. Presl ex DC. } \\
\text { Apocynaceae }\end{array}$ & JJRD 2946; MAGM 192 & $\mathrm{~T} / \mathrm{A}$ \\
\hline h & Asclepias curassavica $\mathrm{L}$. & JL 10 & T/A \\
\hline h & Asclepias glaucescens Kunth & MAGM \& ARC 158 & $\mathrm{~T} / \mathrm{A}$ \\
\hline a & *Mandevilla foliosa (Müll. Arg.) Hemsl. & JJRD 2941 & $\mathrm{~T} / \mathrm{A}$ \\
\hline h & Tabernaemontana tomentosa (Greenm.) A.O. Simões \& M.E.Endress & MAGM \& ARC 221 & T/A \\
\hline h & Telosiphonia hypoleuca (Benth.) & MAGM \& ESC 15 & T/A \\
\hline $\mathbf{r}$ & $\begin{array}{l}\text { *Vallesia spectabilis El. Mey. ex J.F. Morales } \\
\text { Asteraceae }\end{array}$ & MAGM \& ARC 175 & $\mathrm{~T} / \mathrm{A}$ \\
\hline h & *Adenophyllum cancellatum (Cass.) Villarreal & MAGM 113 & $\mathrm{~T} / \mathrm{A}$ \\
\hline $\mathbf{h}$ & *Ageratina cerifera (McVaugh) R.M. King \& H. Rob. & MAGM 191 & $\mathrm{~T} / \mathrm{A}$ \\
\hline h & *Ageratina oligocephala (DC.) R.M. King \& H. Rob. & MAGM 106 & $\mathrm{~T} / \mathrm{A}$ \\
\hline h & *Aldama angustifolia (Hook. \& Arn.) E.E. Schill. \& Panero & MAGM 104 & T/A \\
\hline
\end{tabular}




\begin{tabular}{|c|c|c|c|}
\hline $\mathbf{A}$ & Familia/Especie & Cols./números de colecta & $\mathbf{H} / \mathbf{N}$ \\
\hline $\mathbf{h}$ & Alloispermum scabrum (Lag.) H. Rob. & MAGM 116 & T/A \\
\hline $\mathbf{r}$ & Baccharis pteronioides DC. & MAGM \& ESC 12 & T/A \\
\hline $\mathbf{r}$ & Baccharis salicifolia (Ruiz \& Pav.) Pers. & MAGM \& ANA 86 & T/A \\
\hline $\mathbf{r}$ & Barkleyanthus salicifolius (Kunth) H. Rob. \& Brettell & MAGM 190 & T/A \\
\hline h & Bidens bigelovii var. angustiloba (DC.) Ballard ex Melchert & MAGM 131 & T/A \\
\hline h & Bidens laevis (L.) Britton, Sterns \& Poggenb. & RMcV 26531 & T/A \\
\hline $\mathbf{r}$ & Calea urticifolia (Mill.) DC. & MAGM 145 & T/A \\
\hline h & *Carphochaete grahamii A. Gray & MAGM 102 & T/A \\
\hline h & *Cirsium rhaphilepis (Hemsl.) Petr. & MAGM 200 & T/A \\
\hline h & Cosmos crithmifolius Kunth & MAGM \& ANA 66 & T/A \\
\hline h & Cosmos parviflorus (Jacq.) Pers. & MAGM \& ANA 69 & $\mathrm{~T} / \mathrm{A}$ \\
\hline h & Cosmos sulphureus Cav. & LMVP 12923 & T/A \\
\hline $\mathbf{r}$ & *Critoniopsis autumnalis (McVaugh) H. Rob. & MAGM \& ARC 172 & T/A \\
\hline $\mathbf{a}$ & *Critoniopsis baadii (McVaugh) H. Rob. & MAGM \& ARC 173 & T/A \\
\hline h & Dahlia coccinea Cav. & MAGM \& ESC 2; MAGM 91 & T/A \\
\hline h & *Electranthera cuneifolia (Greenm.) Mesfin, D.J. Crawford \& Pruski & MAGM \& ANA 68,72 & T/A \\
\hline h & *Erigeron velutipes Hook. \& Arn. & MAGM \& ARC 161 & T/A \\
\hline h & Galinsoga parviflora Cav. & MAGM 118 & T/A \\
\hline h & Hieracium pringlei A. Gray & MAGM \& ANA 59, 60, 61 & T/A \\
\hline h & *Hofmeisteria schaffneri (A. Gray) R.M. King \& H. Rob. & MAGM \& ARC 177 & T/A \\
\hline $\mathbf{r}$ & Lagascea helianthifolia Kunth & MAGM \& ARC 166 & T/A \\
\hline h & *Lasianthaea ceanothifolia (Willd.) K.M. Becker & MAGM \& ESC 48 & T/A \\
\hline h & Melampodium perfoliatum (Cav.) Kunth & MAGM \& ESC 56 & $\mathrm{~T} / \mathrm{A}$ \\
\hline h & Pectis uniaristata DC. & MAGM 140 & T/A \\
\hline h & Perityle microglossa Benth. & RMcV 26536 & T/A \\
\hline $\mathbf{a}$ & Podachaenium eminens (Lag.) Sch. Bip. & MAGM 201 & T/A \\
\hline h & *Psacalium megaphyllum (B.L. Rob. \& Greenm.) Rydb. & MAGM \& ESC 23; MAGM 97 & T/A \\
\hline h & *Psacalium palmeri (Greene) H. Rob. \& Brettell & MAGM \& ESC 18 & T/A \\
\hline h & Pseudognaphalium jaliscense (Greenm.) Anderb. & JJRD 2929; MAGM \& DJ 244 & T/A \\
\hline h & *Roldana kerberi (Greenm.) H. Rob. \& Brettell & MAGM 136, 148 & $\mathrm{~T} / \mathrm{A}$ \\
\hline h & *Roldana sessilifolia (Hook. \& Arn.) H. Rob. \& Brettell & MAGM \& FRZ 141 & T/A \\
\hline h & Schkuhria pinnata (Lam.) Kuntze ex Thell. & MAGM \& ARC 233 & T/A \\
\hline h & Stevia ovata Willd. & JJRD 2928; MAGM 111 & T/A \\
\hline h & Stevia viscida Kunth & MAGM 100 & T/A \\
\hline h & Symphyotrichum moranense (Kunth) G.L. Nesom & MAGM \& ARC 180 & $\mathrm{~T} / \mathrm{A}$ \\
\hline h & Tagetes lucida Cav. & MAGM 105 & $\mathrm{~T} / \mathrm{A}$ \\
\hline h & Tagetes micrantha Cav. & MAGM \& ESC 8 & $\mathrm{~T} / \mathrm{A}$ \\
\hline $\mathbf{r}$ & *Verbesina fastigiata B.L. Rob. \& Greenm. & MAGM 146, 147 & T/A \\
\hline $\mathbf{r}$ & *Verbesina pantoptera S.F. Blake & LMVP 12928; MAGM 51 & T/A \\
\hline h & *Vernonanthura serratuloides (Kunth) H. Rob. & MAGM 204 & $\mathrm{~T} / \mathrm{A}$ \\
\hline h & *Vernonia bealliae McVaugh & MAGM 121, 188 & $\mathrm{~T} / \mathrm{A}$ \\
\hline h & *Zinnia angustifolia Kunth & MAGM \& ANA 75 & T/A \\
\hline h & $\begin{array}{l}\text { Zinnia peruviana }(\mathrm{L} .) \mathrm{L} . \\
\text { Begoniaceae }\end{array}$ & JJRD 2944; MAGM 139 & $\mathrm{~T} / \mathrm{A}$ \\
\hline h & Begonia gracilis Kunth & MAGM \& ESC 26, 41 & $\mathrm{~T} / \mathrm{A}$ \\
\hline h & $\begin{array}{l}\text { *Begonia tapatia Burt-Utley \& McVaugh } \\
\text { Bignoniaceae }\end{array}$ & MAGM \& ESC 24 & $\mathrm{~T} / \mathrm{A}$ \\
\hline $\mathbf{a}$ & $\begin{array}{l}\text { Tecoma stans }(\mathrm{L} \text {.) Juss. ex Kunth } \\
\text { Burseraceae }\end{array}$ & MAGM 119 & $\mathrm{~T} / \mathrm{A}$ \\
\hline $\mathbf{a}$ & *Bursera ariensis (Kunth) McVaugh \& Rzed. & MAGM \& ARC 242 & T/A \\
\hline $\mathbf{a}$ & Bursera bipinnata (DC.) Engl. & MAGM et al. 234; MCB 174 & $\mathrm{~T} / \mathrm{A}$ \\
\hline & Bursera fagaroides (Kunth) Engl. & JJRD 2951 & $\mathrm{~T} / \mathrm{A}$ \\
\hline & $\begin{array}{l}\text { *Bursera penicillata (DC.) Engl. } \\
\text { Cactaceae }\end{array}$ & JJRD 2940 & $\mathrm{~T} / \mathrm{A}$ \\
\hline & *Opuntia fuliginosa Griffiths & MAGM \& ESC 13 & T/A \\
\hline
\end{tabular}




\begin{tabular}{|c|c|c|c|}
\hline $\mathbf{A}$ & Familia/Especie & Cols./números de colecta & $\mathbf{H} / \mathbf{N}$ \\
\hline & Campanulaceae & & \\
\hline $\mathbf{h}$ & Lobelia laxiflora Kunth & MAGM et al. 169, 198 & $\mathrm{~T} / \mathrm{A}$ \\
\hline & Caricaceae & & \\
\hline h & *Jarilla heterophylla (Cerv. ex La Llave) Rusby & MAGM \& ARC 219 & $\mathrm{~T} / \mathrm{A}$ \\
\hline h & $\begin{array}{l}\text { *Jarilla nana (Benth.) McVaugh } \\
\text { Cistaceae }\end{array}$ & MAGM \& DJ 246 & $\mathrm{~T} / \mathrm{A}$ \\
\hline $\mathbf{h}$ & $\begin{array}{l}\text { Helianthemum glomeratum (Lag.) Lag. ex Dunal } \\
\text { Convolvulaceae }\end{array}$ & MAGM 143 & $\mathrm{~T} / \mathrm{A}$ \\
\hline h & Ipomoea indica (Burm.) Merr. & MAGM \& ESC 45 & T/A \\
\hline $\mathbf{a}$ & Ipomoea murucoides Roem. \& Schult. & MAGM \& ARC 159 & T/A \\
\hline h & Ipomoea purpurea (L.) Roth & MAGM \& ANA 79 & $\mathrm{~T} / \mathrm{A}$ \\
\hline h & $\begin{array}{l}\text { Ipomoea tricolor Cav. } \\
\text { Crassulaceae }\end{array}$ & MAGM 152 & $\mathrm{~T} / \mathrm{A}$ \\
\hline $\mathbf{h}$ & $\begin{array}{l}\text { Sedum ebracteatum DC. } \\
\text { Cucurbitaceae }\end{array}$ & MAGM 137 & $\mathrm{R} / \mathrm{A}$ \\
\hline b & *Cucurbita radicans Naudin & MAGM \& ESC 49 & $\mathrm{~T} / \mathrm{A}$ \\
\hline b & Cyclanthera multifoliola Cogn. & MAGM \& ESC 36 & $\mathrm{~T} / \mathrm{A}$ \\
\hline b & $\begin{array}{l}\text { Polyclathra cucumerina Bertol. } \\
\text { Ericaceae }\end{array}$ & MAGM 156 & $\mathrm{~T} / \mathrm{A}$ \\
\hline $\mathbf{a}$ & Arbutus xalapensis Kunth & MAGM 211 & $\mathrm{~T} / \mathrm{A}$ \\
\hline a & Arctostaphylos pungens Kunth & JJRD 2900; MAGM 187 & T/A \\
\hline $\mathbf{a}$ & $\begin{array}{l}\text { *Comarostaphylis glaucescens (Kunth) Zucc. ex Klotzsch } \\
\text { Euphorbiaceae }\end{array}$ & MAGM \& ESC 5, 7 & $\mathrm{~T} / \mathrm{A}$ \\
\hline $\mathbf{a}$ & Croton ciliatoglandulifer Ortega & MAGM 114 & $\mathrm{~T} / \mathrm{A}$ \\
\hline h & *Euphorbia sphaerorhiza Benth. & ARC et al. 6189 & $\mathrm{~T} / \mathrm{A}$ \\
\hline h & $\begin{array}{l}\text { *Euphorbia strigosa Hook. \& Arn. } \\
\text { Fabaceae }\end{array}$ & JJRD 2943; MAGM et al. 170 & $\mathrm{~T} / \mathrm{A}$ \\
\hline $\mathbf{r}$ & *Aeschynomene petraea B.L. Rob. & MAGM 183 & T/A \\
\hline h & *Brongniartia cordata McVaugh & MAGM \& MVB 155 & $\mathrm{~T} / \mathrm{A}$ \\
\hline $\mathbf{r}$ & Calliandra grandiflora (L'Hér.) Benth. & MAGM 142 & $\mathrm{~T} / \mathrm{A}$ \\
\hline b & Canavalia villosa Benth. & MAGM 117 & $\mathrm{~T} / \mathrm{A}$ \\
\hline b & Cologania broussonetii (Balb.) DC. & JJRD 2912 & $\mathrm{~T} / \mathrm{A}$ \\
\hline h & Coursetia pumila (Rose) Lavin & MAGM 185 & T/A \\
\hline h & Crotalaria pumila Ortega & MAGM \& ANA 64 & T/A \\
\hline h & Dalea foliolosa (Aiton) Barneby & MAGM \& ESC 10 & T/A \\
\hline h & *Dalea polystachya (Sessé \& Moc.) Barneby & LMTP 4 & $\mathrm{~T} / \mathrm{A}$ \\
\hline h & *Dalea prostrata Ortega & JJRD 2919 & T/A \\
\hline h & Dalea sericea Lag. & MAGM 110 & T/A \\
\hline h & Dalea versicolor Zucc. & JJRD 2911 & T/A \\
\hline h & Desmodium barbatum (L.) Benth. & MAGM \& ESC 32 & $\mathrm{~T} / \mathrm{A}$ \\
\hline h & *Desmodium macrostachyum Hemsl. & JJRD 2908 & $\mathrm{~T} / \mathrm{A}$ \\
\hline h & *Desmodium novogalicianum B.G. Schub. \& McVaugh & JJRD 2914 & $\mathrm{~T} / \mathrm{A}$ \\
\hline h & Desmodium sericophyllum Schltdl. & JJRD 2921 & $\mathrm{~T} / \mathrm{A}$ \\
\hline $\mathbf{h}$ & Desmodium skinneri Benth. ex. Hemsl. & MAGM \& ARC 171 & T/A \\
\hline $\mathbf{r}$ & *Diphysa puberulenta Rydb. & JJRD 2937 & $\mathrm{~T} / \mathrm{A}$ \\
\hline $\mathbf{r}$ & *Eriosema multiflorum B.L. Rob. & MAGM \& ARC 179 & T/A \\
\hline $\mathbf{a}$ & Eysenhardtia polystachya (Ortega) Sarg. & MAGM et al. 237; JJRD 2952 & T/A \\
\hline $\mathbf{r}$ & Indigofera miniata Ortega & JJRD 2916 & T/A \\
\hline $\mathbf{h}$ & Lotus repens (G. Don) Sessé \& Moç. ex Standl. \& Steyerm. & MAGM \& ESC 6 & T/A \\
\hline $\mathbf{a}$ & Lysiloma acapulcense (Kunth) Benth. & MAGM \& MVB 215 & T/A \\
\hline b & Macroptilium gibbosifolium (Ortega) A. Delgado & MAGM \& ANA 99; JJRD 2918 & T/A \\
\hline h & Macroptilium lathyroides (L.) Urb. & MAGM \& ANA 74 & T/A \\
\hline $\mathbf{r}$ & Marina scopa Barneby & ERR 4 & T/A \\
\hline $\mathbf{r}$ & Mimosa aculeaticarpa Ortega & ARC et al. 6196; JJRD 2915 & $\mathrm{~T} / \mathrm{A}$ \\
\hline b & *Nissolia microptera Poir. & JJRD 2917 & T/A \\
\hline
\end{tabular}




\begin{tabular}{|c|c|c|c|}
\hline $\mathbf{A}$ & Familia/Especie & Cols./números de colecta & $\mathbf{H} / \mathbf{N}$ \\
\hline b & Phaseolus anisotrichos Schltdl. & MAGM \& ESC 25 & T/A \\
\hline b & Phaseolus coccineus $\mathrm{L}$. & LMVP 12913 & T/A \\
\hline b & Phaseolus lunatus L. & MAGM \& ARC 163 & $\mathrm{~T} / \mathrm{A}$ \\
\hline b & Phaseolus pauciflorus Sessé \& Moç. ex G. Don & MAGM \& ESC 55 & T/A \\
\hline $\mathbf{a}$ & *Senegalia macilenta (Rose) Britton \& Rose & LMVP 15146 & T/A \\
\hline $\mathbf{r}$ & *Senna foetidissima (Sessé \& Moç. ex G. Don) H.S. Irwin \& Barneby & MAGM \& ANA 85; JJRD 2910 & T/A \\
\hline $\mathbf{r}$ & Senna hirsuta (L.) H.S. Irwin \& Barneby & MAGM \& ARC 160 & T/A \\
\hline $\mathbf{r}$ & Senna septemtrionalis (Viv.) H.S. Irwin \& Barneby & MAGM \& ESC 39 & T/A \\
\hline $\mathbf{r}$ & Vachellia farnesiana (L.) Wight \& Arn. & EHM 5 & T/A \\
\hline $\mathbf{a}$ & Vachellia pennatula (Schltdl. \& Cham.) & JJRD 2958; MAGM \& ESC 12 & T/A \\
\hline h & Zornia reticulata $\mathrm{Sm}$. & JJRD 2920 & T/A \\
\hline h & $\begin{array}{l}\text { Zornia thymifolia Kunth } \\
\text { Fagaceae }\end{array}$ & MAGM \& ESC 4 & $\mathrm{~T} / \mathrm{A}$ \\
\hline a & Quercus castanea Née & MAGM 120; JJRD 2945 & T/A \\
\hline $\mathbf{a}$ & *Quercus eduardi Trel. & JJRD 2902 & T/A \\
\hline a & *Quercus gentryi C.H. Mull. & MAGM \& ARC 228; LMGV 2002 & T/A \\
\hline $\mathbf{a}$ & *Quercus laeta Liebm. & LMGV 2004; MAC \& MAGM 184 & T/A \\
\hline $\mathbf{a}$ & Quercus magnoliifolia Née & MAGM 217; JJRD 2960 & T/A \\
\hline $\mathbf{a}$ & *Quercus resinosa Liebm. & MAGM 186, 209; JJRD 2954 & T/A \\
\hline $\mathbf{a}$ & Quercus salicifolia Née & MAC \& MAGM 185 & T/A \\
\hline a & $\begin{array}{l}\text { *Quercus viminea Trel. } \\
\text { Gesneriaceae }\end{array}$ & JJRD 2956 & T/A \\
\hline h & Achimenes antirrhina (DC.) C.V. Morton & MAGM \& ESC 38 & T/A \\
\hline h & Achimenes erecta (Lam.) H.P. Fuchs & MAGM \& ESC 43 & T/A \\
\hline h & $\begin{array}{l}\text { Achimenes grandiflora (Schltdl.) DC. } \\
\text { Hypericaceae }\end{array}$ & MAGM \& ESC 40 & T/A \\
\hline h & $\begin{array}{l}\text { Hypericum moranense Kunth } \\
\text { Lamiaceae }\end{array}$ & MAGM \& ANA 71 & $\mathrm{~T} / \mathrm{A}$ \\
\hline $\mathbf{r}$ & Hyptis oblongifolia Benth. & JJRD 2945 & $\mathrm{~T} / \mathrm{A}$ \\
\hline h & Hyptis urticoides Kunth & MAGM 124 & T/A \\
\hline h & *Salvia heterotricha Fernald & MAGM \& ESC 6; MAGM 78 & $\mathrm{~T} / \mathrm{A}$ \\
\hline h & *Salvia laevis Benth. & MAGM 199 & T/A \\
\hline h & *Salvia longistyla Benth. & MAGM 149; MAGM \& ARC 282 & T/A \\
\hline h & $\begin{array}{l}* \text { Salvia mexicana } \mathrm{L} \text {. } \\
\text { Lentibulariaceae }\end{array}$ & MAGM \& ANA 84 & T/A \\
\hline h & *Pinguicula oblongiloba A. DC. & MAGM \& ARC 224 & T/A \\
\hline h & $\begin{array}{l}\text { *Pinguicula parvifolia B.L. Rob. } \\
\text { Lythraceae }\end{array}$ & MAGM \& ARC 225 & $\mathrm{~T} / \mathrm{A}$ \\
\hline h & Cuphea llavea Lex. & MAGM \& ESC 17 & T/A \\
\hline h & $\begin{array}{l}\text { Cuphea wrightii A. Gray } \\
\text { Malvaceae }\end{array}$ & MAGM 95; MAGM \& ESC 21 & T/A \\
\hline $\mathbf{r}$ & Malvaviscus arboreus Cav. & MAGM \& ESC 37 & T/A \\
\hline h & Periptera punicea (Lag.) DC. & MAGM 129 & T/A \\
\hline h & $\begin{array}{l}\text { *Triumfetta simplicifolia (Sessé \& Moç.) Fryxell } \\
\text { Nyctaginaceae }\end{array}$ & MAGM 138 & $\mathrm{~T} / \mathrm{A}$ \\
\hline h & $\begin{array}{l}\text { Mirabilis jalapa } \mathrm{L} \text {. } \\
\text { Onagraceae }\end{array}$ & MAGM \& ARC 220 & $\mathrm{~T} / \mathrm{A}$ \\
\hline h & Lopezia racemosa Cav. & MAGM 89, 109 & T/A \\
\hline h & $\begin{array}{l}\text { Oenothera rosea L'Hér. ex Aiton } \\
\text { Orobanchaceae }\end{array}$ & MAGM 196 & T/A \\
\hline h & Agalinis peduncularis (Benth.) Pennell & MAGM 107 & $\mathrm{~T} / \mathrm{M}$ \\
\hline h & Castilleja arvensis Standl. \& Cham. & MAGM 2012 & $\mathrm{~T} / \mathrm{M}$ \\
\hline $\mathbf{h}$ & $\begin{array}{l}\text { Conopholis alpina Liebm. } \\
\text { Oxalidaceae }\end{array}$ & MAGM \& MVB 213 & $\mathrm{~T} / \mathrm{L}$ \\
\hline & Oxalis decaphylla Kunth & ARC et al. 6188; MAGM 240 & T/A \\
\hline
\end{tabular}




\begin{tabular}{|c|c|c|c|}
\hline $\mathbf{A}$ & Familia/Especie & Cols./números de colecta & $\mathbf{H} / \mathbf{N}$ \\
\hline & Passifloraceae & & \\
\hline b & $\begin{array}{l}\text { *Passiflora podadenia Killip } \\
\text { Pineraceae }\end{array}$ & MAGM \& ESC 53 & $\mathrm{~T} / \mathrm{A}$ \\
\hline $\mathbf{r}$ & $\begin{array}{l}\text { *Piper mexicanum (Miq.) DC. } \\
\text { Plantaginaceae }\end{array}$ & MAGM \& ARC 176 & $\mathrm{~T} / \mathrm{A}$ \\
\hline $\mathbf{h}$ & $\begin{array}{l}\text { Russelia coccinea }(\mathrm{L} .) \text { Wettst. } \\
\text { Polemoniaceae }\end{array}$ & MAGM \& MVB 216 & $\mathrm{~T} / \mathrm{A}$ \\
\hline $\mathbf{h}$ & *Loeselia amplectens (Hook. \& Arn.) Benth. ex DC. & MAGM 208; JJRD 2941 & T/A \\
\hline $\mathbf{h}$ & Loeselia ciliata $\mathrm{L}$. & MAGM 101 & T/A \\
\hline $\mathbf{h}$ & $\begin{array}{l}\text { Loeselia glandulosa (Cav.) G. Don } \\
\text { Primulaceae }\end{array}$ & JJRD 2944 & T/A \\
\hline $\mathbf{h}$ & $\begin{array}{l}\text { Anagallis arvensis } \mathrm{L} \text {. } \\
\text { Ranunculaceae }\end{array}$ & MAGM 195 & $\mathrm{~T} / \mathrm{A}$ \\
\hline b & Clematis dioica $\mathrm{L}$. & MAGM 202 & T/A \\
\hline $\mathbf{h}$ & *Delphinium pedatisectum Hemsl. & JJRD 2931 & T/A \\
\hline $\mathbf{h}$ & Ranunculus petiolaris Kunth ex DC. & MAGM \& ARC 238 & $\mathrm{~T} / \mathrm{A}$ \\
\hline $\mathbf{h}$ & $\begin{array}{l}\text { *Thalictrum pubigerum Benth. } \\
\text { Rosaceae }\end{array}$ & MAGM \& ARC 239 & $\mathrm{~T} / \mathrm{A}$ \\
\hline $\mathbf{a}$ & $\begin{array}{l}\text { *Prunus ferruginea Steud. } \\
\text { Rubiaceae }\end{array}$ & MAGM \& ARC 165, 168 & $\mathrm{~T} / \mathrm{A}$ \\
\hline $\mathbf{h}$ & Bouvardia bouvardioides (Seem.) Standl. & MAGM 157 & $\mathrm{~T} / \mathrm{A}$ \\
\hline $\mathbf{h}$ & *Bouvardia chrysantha Mart. & MAGM \& ARC 223 & T/A \\
\hline $\mathbf{h}$ & *Bouvardia scabra Hook. \& Arn. & MAGM 130 & T/A \\
\hline $\mathbf{h}$ & *Bouvardia tenuifolia Standl. & JJRD 2932; MAGM \& ARC 178 & $\mathrm{~T} / \mathrm{A}$ \\
\hline $\mathbf{h}$ & Crusea longiflora (Roem. \& Schult.) W. R. Anderson & JJRD 2947; MAGM \& ANA 87 & $\mathrm{~T} / \mathrm{A}$ \\
\hline $\mathbf{h}$ & $\begin{array}{l}{ }^{*} \text { Crusea psyllioides (Kunth) W. R. Anderson } \\
\text { Santalaceae }\end{array}$ & MAGM \& ANA 77 & T/A \\
\hline $\mathbf{r}$ & Phoradendron brachystachyum (DC.) Oliv. & JJRD 2948; MAGM \& ANA 162 & $\mathrm{H} / \mathrm{M}$ \\
\hline $\mathbf{r}$ & Phoradendron reichenbachianum (Seem.) Oliv. & JJRD 2949 & $\mathrm{H} / \mathrm{M}$ \\
\hline $\mathbf{r}$ & *Phoradendron tetrapterum Krug \& Urb. & MCB 7178 & $\mathrm{H} / \mathrm{M}$ \\
\hline $\mathbf{r}$ & $\begin{array}{l}\text { Phoradendron wattii Krug \& Urb. Greenm. } \\
\text { Sapindaceae }\end{array}$ & MCB 7180 & $\mathrm{H} / \mathrm{M}$ \\
\hline b & $\begin{array}{l}\text { Serjania triquetra Radlk. } \\
\text { Solanaceae }\end{array}$ & MAGM 193 & $\mathrm{~T} / \mathrm{A}$ \\
\hline $\mathbf{r}$ & Cestrum thyrsoideum Kunth & MAGM \& ARC 167 & T/A \\
\hline $\mathbf{r}$ & Jaltomata procumbens (Cav.) J.L. Gentry & MAGM \& ANA 98 & $\mathrm{~T} / \mathrm{A}$ \\
\hline $\mathbf{r}$ & *Lycianthes pringlei (B.L. Rob. \& Greenm.) Bitter & MAGM 128; MAC \& MAGM 181 & T/A \\
\hline $\mathbf{r}$ & Lycianthes moziniana (Dunal) Bitter & MAC \& MAGM 179 & T/A \\
\hline $\mathbf{h}$ & Physalis nicandroides Schltdl. & MAGM 150; MAC \& MAGM 183 & T/A \\
\hline $\mathbf{h}$ & *Physalis orizabae Dunal & MAGM \& ESC 42 & $\mathrm{~T} / \mathrm{A}$ \\
\hline b & Solanum appendiculatum Dunal & MAGM 96; MAGM \& ARC 181 & T/A \\
\hline $\mathbf{r}$ & Solanum diphyllum $\mathrm{L}$. & MAGM \& ARC 235 & $\mathrm{~T} / \mathrm{A}$ \\
\hline $\mathbf{r}$ & Solanum dulcamaroides Dunal & MAGM \& ESC 54 & $\mathrm{~T} / \mathrm{A}$ \\
\hline $\mathbf{r}$ & Solanum ferrugineum Jacq. & MAGM \& ESC 31 & T/A \\
\hline $\mathbf{r}$ & Solanum lanceolatum Cav. & MAGM \& ESC 29, 30 & $\mathrm{~T} / \mathrm{A}$ \\
\hline $\mathbf{r}$ & Solanum pseudocapsicum $\mathrm{L}$. & MAGM \& ARC 218 & $\mathrm{~T} / \mathrm{A}$ \\
\hline $\mathbf{r}$ & Solanum seaforthianum Andrews & MAGM 154 & T/A \\
\hline $\mathbf{h}$ & Solanum stoloniferum Schltdl. \& Bouché & ARC et al. 6190 & $\mathrm{~T} / \mathrm{A}$ \\
\hline $\mathbf{r}$ & $\begin{array}{l}\text { Solanum umbellatum Mill. } \\
\text { Verbenaceae }\end{array}$ & ARC et al. 6191; JJRD 2946 & $\mathrm{~T} / \mathrm{A}$ \\
\hline $\mathbf{r}$ & *Citharexylum mexicanum Moldenke & MAGM \& ARC 174 & $\mathrm{~T} / \mathrm{A}$ \\
\hline $\mathbf{a}$ & $\begin{array}{l}\text { Lippia umbellata Cav. } \\
\text { Ximeniaceae }\end{array}$ & JJRD 2957, 2938 & $\mathrm{~T} / \mathrm{A}$ \\
\hline h & *Ximenia parviflora Benth. & ARC et al. 6192 & $\mathrm{~T} / \mathrm{A}$ \\
\hline
\end{tabular}

